\title{
Graft reconditioning with nitric oxide gas in rat liver transplantation from cardiac death donors.
}

\section{AUTHOR(S):}

Kageyama, Shoichi; Yagi, Shintaro; Tanaka, Hirokazu; Saito, Shunichi; Nagai, Kazuyuki; Hata, Koichiro; Fujimoto, Yasuhiro; Ogura, Yasuhiro; Tolba, Rene; Shinji, Uemoto

\section{CITATION:}

Kageyama, Shoichi ...[et al]. Graft reconditioning with nitric oxide gas in rat liver transplantation from cardiac death donors.. Transplantation 2014, 97(6): 618-625

\section{ISSUE DATE:}

2014-03-27

URL:

http://hdl.handle.net/2433/198289

\section{RIGHT:}

(C) 2014 by Lippincott Williams \& Wilkins; This is not the published version. Please cite only the published version.; この論文は出版社版で ありません。引用の際には出版社版をご確認ご利用ください。 
Graft reconditioning with nitric oxide gas in rat liver transplantation from cardiac death donors

Authors:

Shoichi Kageyama ${ }^{1}$, Shintaro Yagi ${ }^{1}$, Hirokazu Tanaka ${ }^{1}$, Shunichi Saito ${ }^{1}$, Kazuyuki Nagai $^{1,2}$, Koichiro Hata ${ }^{1}$, Yasuhiro Fujimoto ${ }^{1}$, Yasuhiro Ogura ${ }^{1}$, Rene Tolba ${ }^{2}$, Shinji Uemoto $^{1}$

${ }^{1}$ Division of Hepato-Biliary-Pancreatic Surgery and Transplantation, Department of Surgery, Graduate School of Medicine, Kyoto University, Kyoto, Japan

${ }^{2}$ Institute for Laboratory Animal Science and Experimental Surgery, RWTH-Aachen University, Aachen, Germany

Keywords: Cardiac death donor, organ preservation, ischemia-reperfusion injury, warm ischemia, liver transplantation

Word count of abstract: 242 words

Word count of text: 2914 words

Tables: 1

Total figures: 4

Color figures: 3

Address for Correspondence: Shintaro Yagi, MD, PhD Department of Hepatobiliary, Pancreas and Transplant Surgery, Kyoto University, 
54 Kawaharacho, Shogoin, Sakyo-ku, Kyoto, 606-8507, Japan

Tel: +81-75-751-4323; Fax: +81-75-751-4348

E-mail: shintaro@kuhp.kyoto-u.ac.jp

Author contributions:

S.K., S.Y., Y.O., R.T., and S.U. participated in the research design. S.K., S.Y., K.N.,

R.T., and S.U. contributed to the manuscript writing. S.K., S.Y., H.T., S.S., K.N., K.H., Y.F., Y.O., R.T., and S.U. participated in the performance of the research. S.K., S.Y., H.T., S.S., K.N., and S.U. participated in data analysis.

Footnotes:

The authors declare no funding or conflicts of interest. 
Abbreviations:

2-way ANOVA, 2-way analysis of variance; 8-OHdG, 8-hydroxy-2'deoxyguanosine; ALT, alanine aminotransferase; DCD, donation after cardiac death; eNOS, endothelial nitric oxide synthase; ET-1, endothelin-1; IL-6, interleukin-6; iNOS, inducible nitric oxide synthase; I/R injury, ischemia-reperfusion injury; MDA, malondialdehyde; NO, nitric oxide; RECA-1, rat endothelial cell antigen 1; RT-PCR, real-time polymerase chain reaction; SECs, sinusoidal endothelial cells; TNF- $\alpha$, tumor necrosis factor-alpha; VSOP, venous systemic oxygen persufflation. 


\section{Abstract}

Background: Liver transplant outcomes using grafts donated after cardiac death (DCD) remain poor.

Methods: We investigated the effects of ex vivo reconditioning of DCD grafts with venous systemic oxygen persufflation using nitric oxide gas (VSOP-NO) in rat liver transplants. Orthotopic liver transplants were performed in Lewis rats, using DCD grafts prepared using static cold storage alone (group-Control) or reconditioning using VSOP-NO during cold storage (group-VSOP-NO). Experiment I: In a 30-min warm ischemia model, graft damage and hepatic expression of inflammatory cytokines, endothelial nitric oxide synthase (eNOS), inducible nitric oxide synthase (iNOS), and endothelin-1 (ET-1) were examined, and histological analysis was performed 2, 6, 24, and $72 \mathrm{~h}$ after transplantation. Experiment II: In a 60-min warm ischemia model, grafts were evaluated $2 \mathrm{~h}$ after transplantation (6 rats/group), and survival was assessed (7 rats/group).

Results: Experiment I: Group-VSOP-NO had lower alanine aminotransferase (ALT) (P $<0.001)$, hyaluronic acid $(P<0.05)$, and malondialdehyde (MDA) $(P<0.001)$, hepatic interleukin-6 expression (IL-6) $(P<0.05)$, and hepatic tumor necrosis factor-alpha (TNF- $\alpha$ ) expression $(P<0.001)$. Hepatic eNOS expression $(P<0.001)$ was upregulated whereas hepatic iNOS $(P<0.01)$ and ET-1 $(P<0.001)$ expressions were downregulated. Hepatocyte and sinusoidal endothelial cells (SECs) damages were lower in group-VSOP-NO. Experiment II: VSOP-NO decreased ET-1 and 8-hydroxy-2'deoxyguanosine (8-OHdG) expression and improved survival after transplantation by $71.4 \%(P<0.01)$. Conclusion: These results suggest that VSOP-NO effectively reconditions warm ischemia-damaged grafts, presumably by decreasing ET-1 
upregulation and oxidative damage. 


\section{Introduction}

The chronic and growing shortage of donor organs has led to the acceptance of extended criteria donor grafts, such as donations after cardiac death (DCD). Ex vivo graft reconditioning studies of marginal organs using hypothermic (1) and normothermic (2) liver perfusion systems with oxygenation have had promising results. A pilot study demonstrated the safety and feasibility of liver preservation using hypothermic machine perfusion (3); however, normothermic machine perfusion requires additional testing prior to clinical application. Another hypothermic method with a potential to recondition extended criteria donor grafts is venous systemic oxygen persufflation (VSOP), in which grafts are retrogradely perfused with gaseous oxygen through the venous system. VSOP during cold storage has a protective effect against apoptotic changes and improves graft quality, particularly in extended criteria grafts (4-8). Another advantage of VSOP is its ease of use because complex devices are not required, in contrast to machine perfusion systems (4). In VSOP, the use of oxygen alone has yielded inferior results for preserving ischemia-damaged grafts because of the generation of oxygen free radicals (5). Previous studies demonstrated promising results for resuscitating damaged grafts when VSOP was used in combination with antioxidants (9).

Nitric oxide (NO) is a highly diffusible free radical with a short half-life of 5-10 s in biological systems. In the liver, numerous clinical benefits of NO have been described, e.g., positive effects on circulation, energy storage, and mitochondrial damage protection as well as negative effects on oxidative stress and leukocyte activation (10).

Decreased hepatic NO production immediately after portal reperfusion in liver transplantation was suggested to contribute to ischemia-reperfusion (I/R)-dependent 
hepatic injury (11). Some studies showed that administration of NO donors, such as S-nitrosothiols, diazeniumdiolates, sodium nitroprusside, FK409, liver-selective NO donors as well as inhalational NO protect against hepatic I/R injury (10). However, in vivo NO administration after liver transplantation may cause vascular hyporeactivity and decompensation (12). To avoid these, NO administration is limited to liver grafts during storage.

Srinivasan et al. first demonstrated that VSOP using NO gas (VSOP-NO) could decrease portal venous pressure more effectively than VSOP alone in an in vitro study using DCD grafts (13). Yagi et al. confirmed the superiority of VSOP-NO over VSOP alone in in vivo liver transplantation of $30 \%$ small-for-size grafts because of improved microcirculation (14). In contrast to partial graft transplantation, DCD grafts are exposed to severe warm ischemia. Although the precise pathophysiology of warm ischemia-damaged grafts is unknown, the activation of Kupffer cells and CD4+ T cells, the production of oxidative stress, neutrophil-mediated inflammation, and hepatocellular injury are reportedly involved (15). NO is involved in many pathways that affect each other and also have synergistic effects. We hypothesized that the antioxidant effects of NO (16) could protect sinusoidal endothelial cells (SECs), which are involved in early sinusoidal repopulation, consequently improving microcirculation. Thus, we evaluated the effect of VSOP-NO on I/R injury, the recovery phase, and safety in an in vivo rat transplantation model.

\section{Results}

Experiment I. 30-min warm ischemia (Table 1)

Chronological analysis of serum alanine aminotransferase (ALT), hyaluronic acid, 


\section{and malondialdehyde (MDA)}

ALT, a parameter for hepatocellular damage, was significantly lower in group-VSOP-NO over $72 \mathrm{~h}$ [two-way analysis of variance (ANOVA): $P<0.001$ ]. Hyaluronic acid, a marker of endothelial damage (17), was significantly lower in group-VSOP-NO ( $P=0.039)$, and serum MDA, a marker of oxidative stress, was also lower in group-VSOP-NO $(P<0.001)$.

\section{Messenger RNA (mRNA) expression in liver grafts}

Group-VSOP-NO showed downregulated interleukin-6 (IL-6) expression $(P=0.017)$. In addition, in the liver, relative mRNA expressions of tumor necrosis factor-alpha (TNF- $\alpha)(P<0.001)$, endothelin-1 (ET-1) $(P<0.001)$, and inducible nitric oxide synthase (iNOS) were lower in group-VSOP-NO $(P=0.0057)$. Relative mRNA expression of endothelial nitric oxide synthase (eNOS) was significantly higher in group-VSOP-NO $(P<0.001)$.

\section{Histological analysis}

Hematoxylin-eosin staining of livers $6 \mathrm{~h}$ after transplantation (Fig. 1A, B) revealed vacuolization and congestion in group-Control (Fig. 1A), but almost normal liver architecture in group-VSOP-NO (Fig. 1B). Transmission electron microscopy 6 h after transplantation revealed well-preserved SECs covering the space of Disse, which was filled with microvilli (Fig. 1D, 1F) in group-VSOP-NO and partially disrupted sinusoidal walls (Fig. 1C, 1E) in group-Control.

\section{Immunofluorescence of rat endothelial cell antigen-1 (RECA-1)}

Fewer viable RECA-1-positive SECs were observed $6 \mathrm{~h}$ after transplantation in both the groups (Fig. 2B, 2F). Furthermore, $24 \mathrm{~h}$ after transplantation, a few SECs tended to be stained with RECA-1 in group-VSOP-NO alone (Fig. 2C, 2G). Fluorescent SECs were 
also detected $72 \mathrm{~h}$ after transplantation in group-Control (Fig. 2D).

Experiment II-A. 60-min warm ischemia: survival study

In group-Control, the 10-day survival rate was $0 \%$, and all deaths occurred within $30 \mathrm{~h}$ of transplantation (Fig. 3). In group-VSOP-NO, the 10-day survival rate was improved to $71.4 \%(P<0.009)$. Autopsies in group-Control revealed multiple focal necroses $(3.4$ $\pm 0.4)$ in the livers and hemorrhagic ascites. In group-VSOP-NO, there was almost no necrosis $(1.0 \pm 0.0)$.

Experiment II-B. 60-min warm ischemia: evaluation of early phase injury

Relative hepatic ET-1 mRNA expression levels 2 h after portal reperfusion were higher in group-Control than in group-VSOP-NO $(P<0.005$; Fig. 4A). Figure 4B and $\mathrm{C}$ show the staining of SECs with 8-hydroxy-2'deoxyguanosine (8-OHdG) as a marker for oxidative DNA damage $2 \mathrm{~h}$ after portal reperfusion. In group-Control (Fig. 4B), broad sinusoidal cells and partial hepatocytes were stained, whereas in group-VSOP-NO, few sinusoidal cells were stained (Fig. 4C).

\section{Discussion}

We report, for the first time, the beneficial effects of VSOP-NO in in vivo rat DCD liver transplantation. The changes in severe $\mathrm{I} / \mathrm{R}$ injury associated with warm ischemia-damaged DCD grafts reportedly occur in two distinct phases i.e., the early phase $<2 \mathrm{~h}$ after portal reperfusion and the later injury phase 6-48 $\mathrm{h}$ after portal reperfusion (16). To evaluate how hepatocytes and SECs are affected in DCD transplantation, we assessed inflammatory cytokines, oxidative stress, and indicators of hepatocyte and SEC function as well as parameters influenced by NO administration, including ET-1, iNOS, and eNOS. We chose $2 \mathrm{~h}$ as the early phase, 6-24 h as the late 
phase, and $72 \mathrm{~h}$ as the early recovery period associated with I/R injury.

In Experiment I, VSOP-NO decreased ET-1 and iNOS expression levels $2 \mathrm{~h}$ after portal reperfusion and attenuated increases in ALT, inflammatory cytokines, eNOS upregulation, and MDA elevation 6-24 h after portal reperfusion. The early phase of warm ischemia-induced reperfusion injury in DCD grafts is reportedly associated with activation of Kupffer cells and production of oxidative stress. Moreover, Klune et al. reported that the first step of the I/R injury cascade is the liberation of ET-1, which activates stellate cells and leads to constriction of hepatic sinusoids and accumulation of oxidative stress, activating Kupffer cells and initiating upregulation of inflammatory cytokines (18). In Experiment II-A, death occurred $2 \mathrm{~h}$ after portal reperfusion, and autopsies of both groups suggested that the cause was graft failure without vascular or biliary complications or bleeding due to gaseous perfusion, which has been associated with VSOP. We concluded that differences at $2 \mathrm{~h}$ after portal reperfusion influenced the survival rate in Experiment II-A.

According to the results of real-time polymerase chain reaction (RT-PCR) in Experiment I, the key parameters affecting survival are ET-1 and iNOS. However, exogenous NO may have suppressed iNOS in the current study; therefore, we focused on ET-1. In Experiment I, we used MDA, an end-product of lipid peroxidation, to assess whether oxidative stress plays a role in the effect of VSOP-NO on I/R injury. The spectrophotometric thiobarbituric acid-reactive substances assay used in the current study is extremely easy to use, but is nonspecific; other substrates (e.g., aldehydes) can react with thiobarbituric acid (19). By contrast, 8-OHdG is a sensitive indicator of oxidative DNA damage. To determine if oxidative stress is an early factor in survival, 8-OHdG was used in Experiment II. We speculate that the critical benefit of VSOP-NO 
administration is the decrease in oxidative damage and ET-1 upregulation, resulting in inactivation of inflammatory responses and protection of SECs and hepatocytes.

In our study, VSOP-NO decreased the incidence of initial death due to long warm ischemia. Several studies have demonstrated the relationship between the duration of warm ischemia and post-transplant survival in pig (20) and rat (21) models. We chose 60 min as the critical time for prolonged warm ischemia because a 1-week survival rate of $<10 \%$ is appropriate for primary nonfunction, in which we observed decreased oxidative damage and ET-1 upregulation, which we consider key effects of VSOP-NO. In the liver, iNOS is not expressed under normal circumstances; however, under certain conditions, iNOS has either a protective or toxic effect (22). In hepatic I/R injury, iNOS exacerbates liver injury (23). In addition, iNOS expression is known to be downregulated by steroids, tumor growth factor- $\beta$, the heat shock response, $\mathrm{p} 53$, and NO itself (24). In this study, exogenous NO decreased iNOS upregulation and suppressed the exacerbation of hepatic I/R injury. However, eNOS is constitutively expressed in SECs and is related to sinusoidal circulation (25). A previous report demonstrated that repopulation of SECs requires 12-48 h in denuded sinusoids (26). Our results demonstrated eNOS upregulation $24 \mathrm{~h}$ after transplantation. Shear stress has been reported as an inducer of eNOS production (27). In the current model, warm ischemia strongly injured SECs. In Experiment II-A, all parameters but one in group-Control approached those in group-VSOP-NO by day 3 and no rats died. Our results demonstrate that 30 -min warm ischemia is not crucial, and thus hepatocyte damage could recover by 3 days. Hyaluronic acid, a parameter of sinusoidal endothelial injury, showed a different trend from the other parameters, increasing 6-24 h after portal reperfusion but not recovering to the level in group-VSOP-NO within $72 \mathrm{~h}$. On 
the other hand, RECA-1 immunohistochemical analysis demonstrated that damaged SECs recovered within $24 \mathrm{~h}$ of transplantation in group-VSOP-NO and within $72 \mathrm{~h}$ in group-Control. We hypothesize that VSOP-NO stimulated the early repopulation of the endothelial structure, resulting in eNOS upregulation $24 \mathrm{~h}$ after portal reperfusion, but the recovery of hyaluronic acid indicates that the SECs require $>72 \mathrm{~h}$ for adequate functional recovery.

After NO administration, excess NO promotes its reaction with superoxide (28), resulting in the formation of peroxynitrite (29), a molecule extremely toxic to cells (30). We previously demonstrated NO concentration of 40 ppm was optimal to produce a significant effect (14). This study demonstrated the safety of NO administration during cold storage.

Remarkably, in Experiment II-A, VSOP-NO resulted in successful reconditioning of severely damaged DCD grafts. A few studies have reported reclamation of DCD grafts exposed to 60-min warm ischemia. Peaks et al. and Tolboom et al. successfully rescued almost all transplants that underwent 60-min warm ischemia by normothermic extracorporeal perfusion $(2,31)$. In terms of survival, after 60 -min warm ischemia, VSOP-NO appeared to be inferior to machine perfusion. The insult in DCD grafts is multifactorial and depends on the duration of warm ischemia as well as recipient status, anhepatic time, I/R injury involving preservation solutions, duration of cold ischemia, and surgical techniques. Stegemann et al. previously reported the superiority of VSOP over machine perfusion in a model of long-term preservation of DCD grafts (32). Further studies are needed to compare normothermic machine perfusion and VSOP-NO. Machine perfusion requires a pump, plasma, erythrocytes, and a dialyzer, whereas the VSOP system requires only gas cylinders and ice. Despite efforts to simplify and 
decrease expenses associated with machine perfusion, the current VSOP system remains more convenient and economical.

In the current study, we applied 3-h cold ischemia, which is shorter than that for DCD transplantation in the typical clinical setting. DCD grafts exposed to prolonged warm ischemia poorly tolerated longer durations of cold ischemia (33).

Other methods to recover liver grafts, such as hypothermic or normothermic machine perfusion and VSOP, have been shown to be effective for longer periods of storage, but not yet in combination with longer warm ischemia and following longer cold ischemia. In future studies, we plan to evaluate a prolonged cold ischemia group to demonstrate the efficacy of VSOP-NO for DCD grafts. Tolboom et al. reported that normothermic oxygenated perfusion results in less $\mathrm{I} / \mathrm{R}$ injury (2). Thus, we speculate that subnormothermic retrograde perfusion preservation with gaseous NO could provide improved graft survival.

Other clinically relevant long-term effects of warm ischemia, such as ischemic cholangiopathy, were not investigated in this study. Cholangiography and quantitative comparisons require long-term observations (34); thus, further studies involving long-term observations of DCD in a large animal model are needed.

In conclusion, retrograde administration of gaseous oxygen and NO during cold storage is an easy and safe method to recondition marginal DCD liver grafts. Our results will benefit future applications of this method; however, we recommend that our technique should applied cautiously to the clinical setting. Specifically, further experiments with large animals and longer observation periods are necessary prior to applying this novel organ preservation technique to clinically marginal grafts. 


\section{Material and Methods}

\section{Animals}

All experiments were performed in accordance with the Animal Research Committee of Kyoto University, and all animals received humane care according to criteria outlined in the "Guide for the Care and Use of Laboratory Animals" prepared by the National Academy of Sciences and published by the National Institutes of Health (NIH Publication No 86-23, revised in 1985). Male LEW/CrlCrlj rats weighting 180-220 g (Charles River Laboratories Japan, Inc., Yokohama, Japan) were used as liver donors/recipients. The animals were housed under specific pathogen-free conditions in a temperature- and humidity-controlled environment with a 12-h light/dark cycle with access to tap water and standard chow pellets ad libitum.

\section{Donor procedure and graft storage}

\section{Experiment I. 30-min warm ischemia}

Rat livers were retrieved after 30-min warm ischemia induced by phrenotomy 5 min after injection of 300 units of heparin sodium, as described previously (21). In group-Control, grafts were preserved in University of Wisconsin solution (ViaSpan, Astellas Pharma Inc., Tokyo, Japan) at $4^{\circ} \mathrm{C}$. In group-VSOP-NO, grafts were treated by VSOP using gaseous NO at $40 \mathrm{ppm}$, as previously reported (14). Briefly, catheter was inserted into the thoracic caval vein and both the portal and infrahepatic caval veins were clamped with atraumatic clips. Small pinpricks were made which allowed the gaseous oxygen and NO from the catheter to escape during cold storage. After $3 \mathrm{~h}$ of cold storage, orthotopic liver transplantation was performed using the DCD grafts and a modified Kamada's method (35). Anhepatic time was $14.0 \pm 0.3 \mathrm{~min}$ in group-VSOP-NO and $14.3 \pm 0.2 \mathrm{~min}$ in group-Control $(P=0.38)$. 
Subsequently, 2, 6, 24, and $72 \mathrm{~h}$ after portal reperfusion $(\mathrm{n}=6$ at each time point for each group), serum and liver graft tissue samples were collected prior to euthanasia by exsanguination.

\section{Experiment II-A. 60-min warm ischemia: survival study}

The lethality of a longer-duration (60-min) warm ischemia model and the effect of VSOP-NO against lethal stress were verified in a series of experiments. Rats were divided into group-Control and group-VSOP-NO ( $\mathrm{n}=7$ each). The 60-min warm ischemia-damaged grafts were transplanted into recipients similar to Experiment I. Survival rate was investigated for 10 days after transplantation. To determine histological alterations, necrotic lesions were graded on a 4-point scale as follows: $1=$ no changes or negligible lesions, affecting $0 \%-10 \%$ of field, $2=$ mild, affecting $10 \%-$ $40 \%, 3=$ moderate, affecting $40 \%-70 \%$, and $4=$ severe, affecting $>70 \%$ ).

\section{Experiment II-B. 60-min warm ischemia: evaluation of early injury}

The relationship between the results of Experiments I and II-A was confirmed in another series of experiments. Group-Control and group-VSOP-NO ( $\mathrm{n}=6$ each) rats underwent the same surgery as animals in Experiment II-A. Liver graft tissues were sampled $2 \mathrm{~h}$ after portal reperfusion.

\section{Measurement of liver enzymes}

Serum ALT and hyaluronic acid levels were measured by a standard spectrophotometric method using an automated clinical analyzer (JCA-BM9030, JEOL Ltd., Tokyo, Japan).

\section{Malondialdehyde}

To estimate the levels of lipid peroxidation products, serum MDA levels were measured using the thiobarbituric acid assay according to the manufacturer's protocol (NWLSS ${ }^{\mathrm{TM}}$ Malondialdehyde Assay, Northwest Life Science Specialties, LLC, WA, USA). 


\section{Real-Time Polymerase Chain Reaction}

For gene expression analysis, RT-PCR was performed using TaqMan ${ }^{\circledR}$ technology, as previously reported (36). The probe and primers for TNF- $\alpha$ (assay ID Rn01525859_g1), IL-6 (assay ID Rn01410330_m1), iNOS (assay ID Rn00561646_m1), eNOS (assay ID Rn02132634_s1), ET-1 (assay ID Rn00561129_m1), and beta-actin ( $\beta$-actin) (assay ID Rn00667869_m1) were obtained for $\operatorname{TaqMan}^{\circledR}$ gene expression assays from Applied Biosystems, Life Technologies Japan Ltd., Japan.

\section{Immunohistochemistry}

To assess the damage and recovery of SECs, immunofluorescence was assessed using rat endothelial cell antigen 1 (RECA-1: MCA-970R, Serotec, Oxford, UK). Alexa Fluor® 594 goat anti-mouse IgG (A-110005, Invitrogen, Life Technologies Japan Ltd., Japan) was used as the secondary antibody. To evaluate oxidative DNA damage, 8-OHdG (N45.1, Japan Institute for the Control of Aging, Nikken Seil Co., Ltd, Shizuoka, Japan) and hematoxylin were used according to the manufacturer's protocol.

\section{Histological assessment of tissue injury}

Liver samples were fixed with 4\% paraformaldehyde, embedded in paraffin wax, and sectioned (4- $\mu$ m thickness). Slides were stained with hematoxylin-eosin.

\section{Electron microscopy}

Fixation of liver samples was performed as previously reported (37). Sections were observed by transmission electron microscope (H-7650: Hitachi, Ltd., Tokyo, Japan).

\section{Statistical analyses}

Results are expressed as mean \pm standard error for each group. Statistical analysis of the groups at each time point was performed with two-way ANOVA and Bonferroni's post-hoc test. For the survival study, a Mantel-Cox log-rank analysis was performed. 
The Mann-Whitney $U$-test was used to analyze hepatic ET-1 expression in Experiment II-B. $P$ of $<0.05$ was considered statistically significant. Calculations were performed with Prism Software Version 5.0c (GraphPad Software, Inc. CA, USA). 


\section{Acknowledgments}

This work was supported by a Grant-in-Aid from the Japan Society for the Promotion of Science, Tokyo, Japan (No. 60447969).

The authors thank Keiko Furuta and Hiroshi Kameda for their technical assistance with the electron microscopy studies.

The authors would like to thank Enago (www.enago.jp) for the English language review. 


\section{References}

1. Dutkowski P, Furrer K, Tian Y, Graf R, Clavien PA. Novel short-term hypothermic oxygenated perfusion (HOPE) system prevents injury in rat liver graft from non-heart beating donor. Ann Surg 2006; 244: 968.

2. Tolboom H, Pouw RE, Izamis ML, et al. Recovery of warm ischemic rat liver grafts by normothermic extracorporeal perfusion. Transplantation 2009; 87: 170.

3. Guarrera JV, Henry SD, Samstein B, et al. Hypothermic machine preservation in human liver transplantation: the first clinical series. Am J Transplant 2010; 10: 372.

4. Minor T, Klauke H, Nagelschmidt M, Isselhard W. Reduction of proteolysis by venous-systemic oxygen persufflation during rat liver preservation and improved functional outcome after transplantation. Transplantation 1997; 63: 365.

5. Minor T, Kotting M. Gaseous oxygen for hypothermic preservation of predamaged liver grafts: fuel to cellular homeostasis or radical tissue alteration? Cryobiology 2000; 40: 182.

6. Tolba RH, Schildberg FA, Schnurr C, Glatzel U, Decker D, Minor T. Reduced liver apoptosis after venous systemic oxygen persufflation in non-heart-beating donors. J Invest Surg 2006; 19: 219.

7. Treckmann J, Minor T, Saad S, et al. Retrograde oxygen persufflation preservation of human livers: a pilot study. Liver Transpl 2008; 14: 358.

8. Minor T, Koetting M, Kaiser G, Efferz P, Luer B, Paul A. Hypothermic reconditioning by gaseous oxygen improves survival after liver transplantation in the pig. Am J Transplant 2011; 11: 2627.

9. Sun HW, Shen F, Zhou YM. Influence of perfusion by gaseous oxygen persufflation on rat donor liver. Hepatobiliary Pancreat Dis Int 2006; 5: 195. 
10. Abu-Amara M, Yang SY, Seifalian A, Davidson B, Fuller B. The nitric oxide pathway--evidence and mechanisms for protection against liver ischaemia reperfusion injury. Liver Int 2012; 32: 531.

11. Varadarajan R, Golden-Mason L, Young L, et al. Nitric oxide in early ischaemia reperfusion injury during human orthotopic liver transplantation. Transplantation 2004; 78: 250.

12. Thiemermann C, Szabo C, Mitchell JA, Vane JR. Vascular hyporeactivity to vasoconstrictor agents and hemodynamic decompensation in hemorrhagic shock is mediated by nitric oxide. Proc Natl Acad Sci U S A 1993; 90: 267.

13. Srinivasan PK, Yagi S, Doorschodt B, et al. Impact of venous systemic oxygen persufflation supplemented with nitric oxide gas on cold-stored, warm ischemia-damaged experimental liver grafts. Liver Transpl 2012; 18: 219.

14. Yagi S, Nagai K, Kadaba P, et al. A novel organ preservation for small partial liver transplantations in rats: venous systemic oxygen persuf fl ation with nitric oxide gas. Am J Transplant 2013; 13: 222.

15. Klune JR, Tsung A. Molecular biology of liver ischemia/reperfusion injury: established mechanisms and recent advancements. Surg Clin North Am 2010; 90: 665. 16. Stamler JS. Redox signaling: nitrosylation and related target interactions of nitric oxide. Cell 1994; 78: 931.

17. Schon MR, Kollmar O, Wolf S, et al. Liver transplantation after organ preservation with normothermic extracorporeal perfusion. Ann Surg 2001; 233: 114. 18. Busuttil RW, Tanaka K. The utility of marginal donors in liver transplantation. Liver Transpl 2003; 9: 651.

19. Mayne ST. Antioxidant nutrients and chronic disease: use of biomarkers of 
exposure and oxidative stress status in epidemiologic research. $J$ Nutr 2003; 133 Suppl 3: 933S.

20. Monbaliu D, van Pelt J, De Vos R, et al. Primary graft nonfunction and Kupffer cell activation after liver transplantation from non-heart-beating donors in pigs. Liver Transpl 2007; 13: 239.

21. He XS, Ma Y, Wu LW, et al. Safe time to warm ischemia and posttransplant survival of liver graft from non-heart-beating donors. World J Gastroenterol 2004; 10: 3157.

22. Li J, Billiar TR. Nitric Oxide. IV. Determinants of nitric oxide protection and toxicity in liver. Am J Physiol 1999; 276: G1069.

23. Lee VG, Johnson ML, Baust J, Laubach VE, Watkins SC, Billiar TR. The roles of iNOS in liver ischemia-reperfusion injury. Shock 2001; 16: 355.

24. Taylor BS, Alarcon LH, Billiar TR. Inducible nitric oxide synthase in the liver: regulation and function. Biochemistry (Mosc) 1998; 63: 766.

25. Shah V, Haddad FG, Garcia-Cardena G, et al. Liver sinusoidal endothelial cells are responsible for nitric oxide modulation of resistance in the hepatic sinusoids. $J$ Clin Invest 1997; 100: 2923.

26. Stolz DB, Ross MA, Ikeda A, et al. Sinusoidal endothelial cell repopulation following ischemia/reperfusion injury in rat liver transplantation. Hepatology 2007; 46: 1464.

27. Fleming I, Bauersachs J, Fisslthaler B, Busse R. Ca2+-independent activation of the endothelial nitric oxide synthase in response to tyrosine phosphatase inhibitors and fluid shear stress. Circ Res 1998; 82: 686.

28. Ferdinandy P, Schulz R. Nitric oxide, superoxide, and peroxynitrite in 
myocardial ischaemia-reperfusion injury and preconditioning. Br J Pharmacol 2003; 138: 532.

29. Song SW, Tolba RH, Yonezawa K, Manekeller S, Minor T. Exogenous superoxide dismutase prevents peroxynitrite-induced apoptosis in non-heart-beating donor livers. Eur Surg Res 2008; 41: 353.

30. Hon WM, Lee KH, Khoo HE. Nitric oxide in liver diseases: friend, foe, or just passerby? Ann N Y Acad Sci 2002; 962: 275.

31. Perk S, Izamis ML, Tolboom H, et al. A metabolic index of ischemic injury for perfusion-recovery of cadaveric rat livers. PLoS One 2011; 6: e28518.

32. Stegemann J, Hirner A, Rauen U, Minor T. Gaseous oxygen persufflation or oxygenated machine perfusion with Custodiol-N for long-term preservation of ischemic rat livers? Cryobiology 2009; 58: 45.

33. Monbaliu D, Liu Q, Vekemans K, Roskams T, Pirenne J. Potentiation of adverse effects of cold by warm ischemia in circulatory death donors for porcine liver transplantation. Transplant Proc 2012; 44: 2874.

34. Imamura H, Rocheleau B, Cote J, Huet PM. Long-term consequence of rat orthotopic liver transplantation with and without hepatic arterial reconstruction: a clinical, pathological, and hemodynamic study. Hepatology 1997; 26: 198.

35. Kamada N, Calne RY. Orthotopic liver transplantation in the rat. Technique using cuff for portal vein anastomosis and biliary drainage. Transplantation 1979; 28 : 47.

36. Nagai K, Yagi S, Afify M, Bleilevens C, Uemoto S, Tolba RH. Impact of venous-systemic oxygen persufflation with nitric oxide gas on steatotic grafts after partial orthotopic liver transplantation in rats. Transplantation 2013; 95: 78. 
37. Yamanaka K, Hatano E, Narita M, et al. Olprinone attenuates excessive shear stress through up-regulation of endothelial nitric oxide synthase in a rat excessive hepatectomy model. Liver Transpl 2011; 17: 60. 
Table 1. Time-course for serum analysis and gene expression analysis

\begin{tabular}{cccccc}
\hline & & 2 hour & 6 hour & 1 day & 3 day \\
\hline ALT & Control & $2770.0 \pm 151.3$ & $4084.2 \pm 1196.5$ & $1895.8 \pm 303.0$ & $234.0 \pm 92.8$ \\
$(\mathrm{IU} / \mathrm{ml})$ & VSOP-NO & $2035.0 \pm 261.2$ & $1088.5 \pm 261.2^{* * *}$ & $457.3 \pm 43.0$ & $205.5 \pm 92.7$ \\
\hline Hyaluronic Acid & Control & $294.5 \pm 90.8$ & $281.6 \pm 58.8$ & $1179.9 \pm 202.1$ & $428.2 \pm 133.6$ \\
$(\mathrm{ng} / \mathrm{ml})$ & VSOP-NO & $197.2 \pm 12.5$ & $117.2 \pm 19.5$ & $695.1 \pm 174.7$ & $118.6 \pm 18.3$ \\
\hline MDA & Control & $4.8 \pm 0.8$ & $7.7 \pm 0.5$ & $4.2 \pm 0.9$ & $2.1 \pm 0.3$ \\
$(\mu \mathrm{M})$ & VSOP-NO & $4.0 \pm 0.7$ & $2.8 \pm 0.5 * * *$ & $1.9 \pm 0.3 *$ & $2.6 \pm 0.3$ \\
\hline \hline IL-6 & Control & $2098.7 \pm 1996.7$ & $4227.4 \pm 960.0$ & $25.6 \pm 8.9$ & $43.9 \pm 20.0$ \\
$(\% \beta$-actin $)$ & VSOP-NO & $85.2 \pm 31.6$ & $753.9 \pm 185.7 *$ & $1.8 \pm 0.3$ & $29.4 \pm 11.8$ \\
\hline TNF $\alpha$ & Control & $40.6 \pm 12.2$ & $115.1 \pm 21.8$ & $31.0 \pm 6.1$ & $18.4 \pm 2.3$ \\
$(\% \beta$-actin $)$ & VSOP-NO & $21.9 \pm 7.9$ & $23.1 \pm 8.9 * * *$ & $18.5 \pm 5.7$ & $22.4 \pm 6.2$ \\
\hline ET-1 & Control & $16.0 \pm 5.6$ & $21.0 \pm 2.9$ & $8.0 \pm 1.0$ & $1.7 \pm 0.3$ \\
$(\% \beta$-actin $)$ & VSOP-NO & $3.1 \pm 0.6 * *$ & $6.1 \pm 0.9 * * *$ & $1.4 \pm 0.3$ & $1.7 \pm 0.3$ \\
\hline iNOS & Control & $7.1 \pm 2.4$ & $4.4 \pm 1.0$ & $2.7 \pm 1.4$ & $3.2 \pm 0.8$ \\
$(\% \beta$-actin $)$ & VSOP-NO & $1.4 \pm 0.3 * *$ & $2.4 \pm 0.7$ & $2.3 \pm 0.8$ & $1.7 \pm 0.5$ \\
\hline eNOS & Control & $0.3 \pm 0.1$ & $1.0 \pm 0.2$ & $0.6 \pm 0.1$ & $1.0 \pm 0.1$ \\
\hline VSOP-NO & $1.0 \pm 0.2$ & $1.5 \pm 0.5$ & $4.6 \pm 0.4 * * *$ & $1.4 \pm 0.3$ \\
\hline
\end{tabular}

Mean \pm standard error of the mean of serum value after transplantation. ALT, alanine aminotransferase; MDA, malondialdehyde; IL-6, interleukin-6; TNF $\alpha$, tumor necrosis factor alpha; ET-1, endothelin-1; iNOS, inducible nitric oxide synthase; eNOS, endothelial nitric oxide synthase; Control, graft stored in simple cold storage; VSOP-NO, graft stored in venous systemic oxygen persufflation with nitric oxide gas. $p<0.001$ in AST, $p=0.039$ in hyaluronic acid, $p<0.001$ in MDA, $p=0.017$ in IL-6, $p<0.001$ in TNF $\alpha, p<0.001$ in ET-1, $p=0.0057$ in iNOS, and $p<0.001$ in eNOS. Bonferroni's post-test: $* p<0.05,{ }^{* *} p<0.01$, and ${ }^{* * *} p<0.001$ vs VSOP-NO. 


\section{Figure Legends}

\section{Figure 1}

Histological analysis using hematoxylin-eosin staining (A, B; original magnification $\times 100)$ and ultrastructural analysis using transmission electron microscopy (C-F) of the hepatic microstructure 6 h after liver transplantation in Control (A, C, E) and VSOP-NO $(\mathrm{B}, \mathrm{D}, \mathrm{F})$ groups. Black bars in hematoxylin-eosin images indicate $100 \mu \mathrm{m}(\mathrm{A}, \mathrm{B})$, and black bars in transmission electron microscopy images indicate $2 \mu \mathrm{m}(\mathrm{C}, \mathrm{D})$ and $500 \mathrm{~nm}$ (E, F).

$\mathrm{CV}$, central venous area; PV, portal venous area; HC, hepatocytes; SEC, sinusoidal endothelial cells; Control grafts stored in cold storage alone; VSOP-NO grafts stored in venous systemic oxygen persufflation using nitric oxide gas. Sinusoidal epithelial cell (SEC) destruction (arrow) was observed.

\section{Figure 2}

Immunofluorescence analysis using rat endothelial cell antigen 1 (RECA-1) of hepatic expression 2, 6, 24, and $72 \mathrm{~h}$ after portal reperfusion in Control (A-D) and VSOP-NO (E-H) groups. CV, central venous area; PV, portal venous area; Control grafts stored in cold storage alone; VSOP-NO grafts stored in venous systemic oxygen persufflation using gaseous NO.

\section{Figure 3}

Kaplan-Meier survival curves of recipient rats after transplantation of 60 -min warm ischemia-damaged livers stored in cold storage alone (Control) and venous systemic oxygen persufflation using nitric oxide gas (VSOP-NO). Survival rates for VSOP-NO 
and Control were $71.4 \%$ and $14.3 \%$, respectively, at $12 \mathrm{~h}$ and $71.4 \%$ and $0 \%$, respectively, at 10 days; $P=0.009$ vs. Control using the log-rank test ( $n=6$ per group).

\section{Figure 4}

Relative messenger RNA expression in the liver for endothelin-1 (ET-1) $2 \mathrm{~h}$ after transplantation of 60-min warm ischemia-damaged grafts in group-Control and groupvenous systemic oxygen persufflation using nitric oxide gas (VSOP-NO) (A). The Mann-Whitney $U$-test was used. ${ }^{* *} P<0.01$ vs. Control (mean \pm standard error of mean, $\mathrm{n}=6$ per group). Immunofluorescence using 8-hydroxy-2'-deoxyguanosine (8-OHdG) and hematoxylin for hepatic expression $2 \mathrm{~h}$ after liver transplantation of 60-min warm ischemia-damaged grafts in group-Control (B) and group-VSOP-NO (C). The black bars indicate a length of $100 \mathrm{~mm}$. CV, central venous area; PV, portal venous area; Control grafts stored in cold storage alone; VSOP-NO grafts stored in venous systemic oxygen persufflation with nitric oxide gas. 


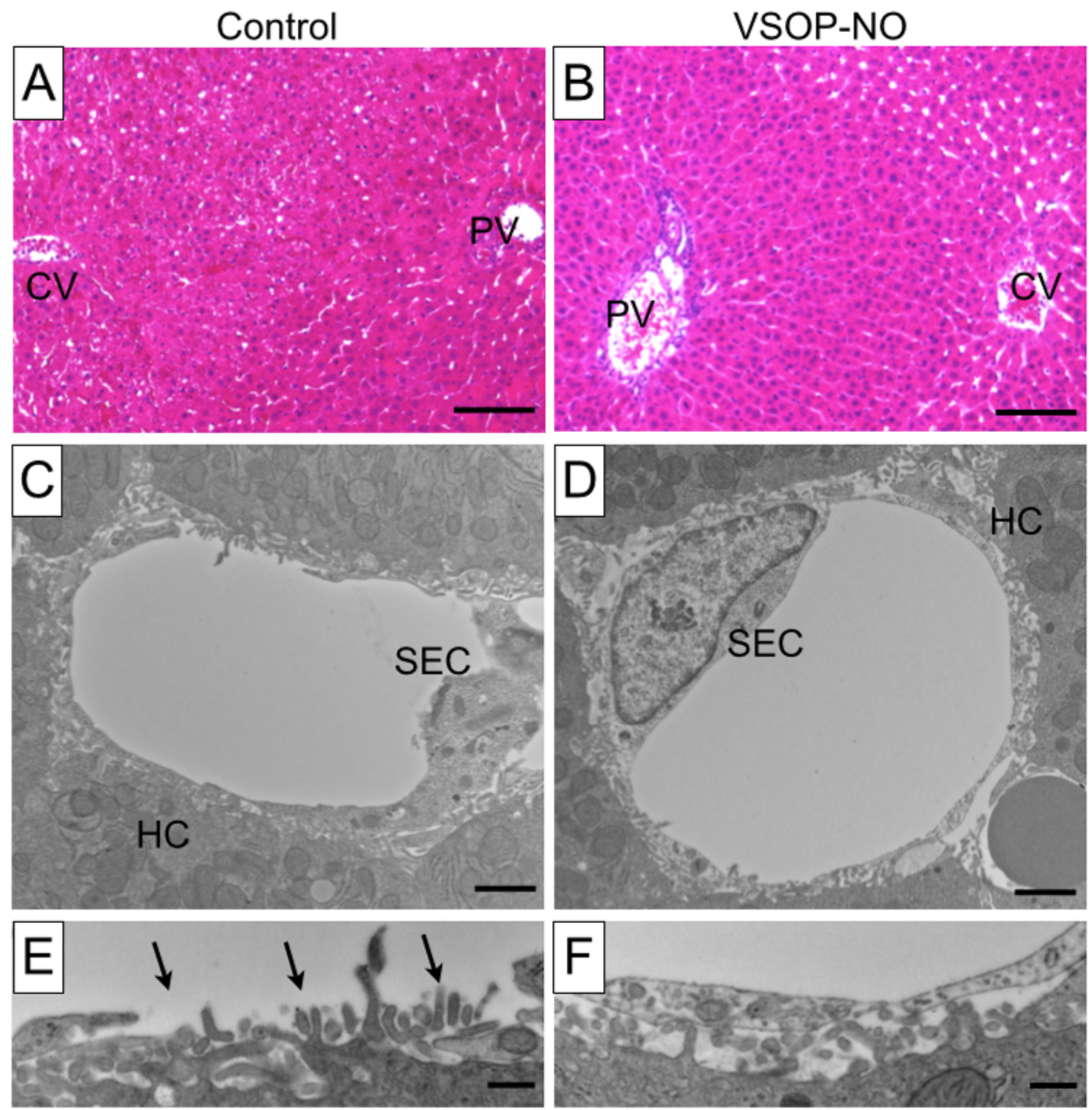

Figure 1 


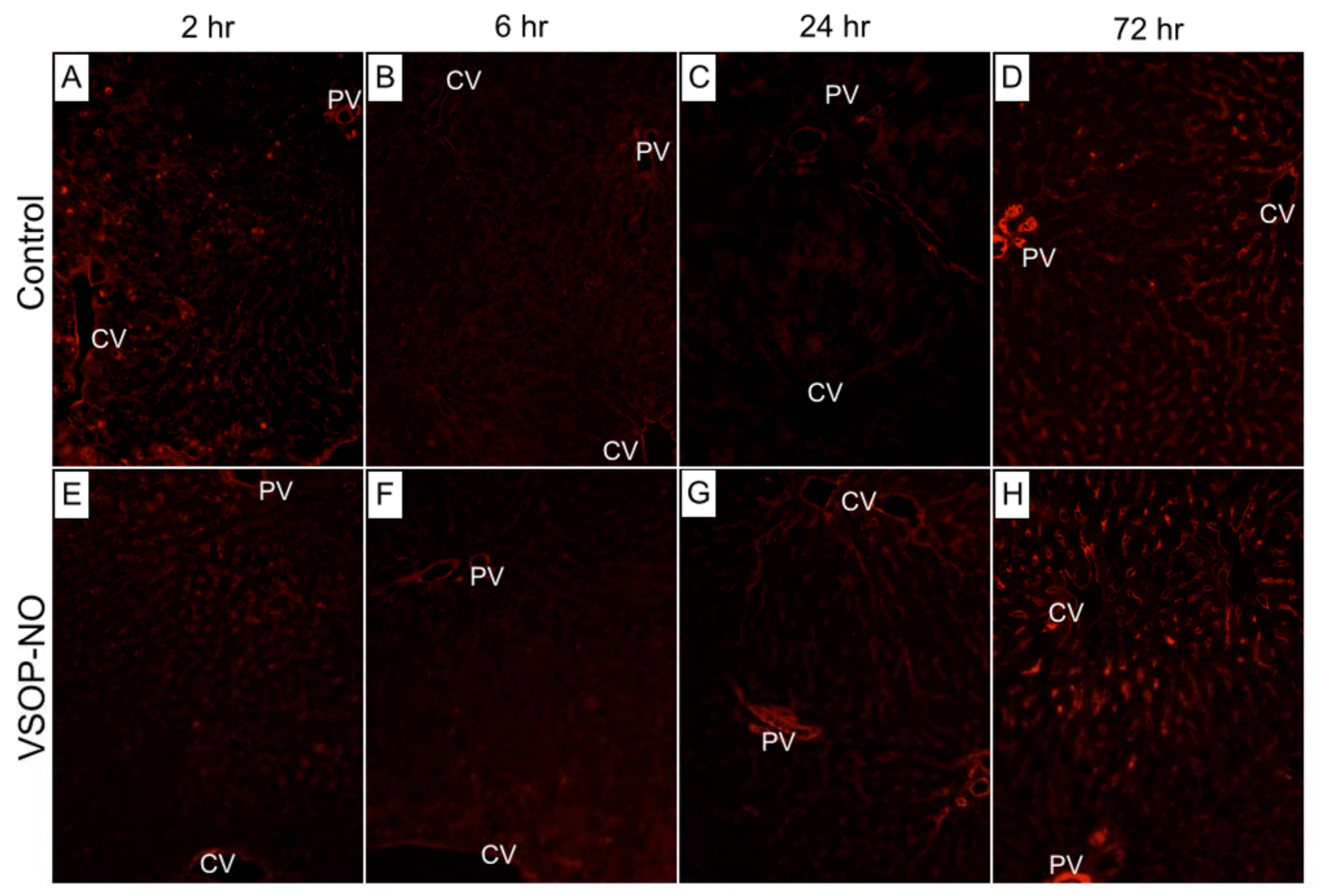

RECA-1

Figure 2 


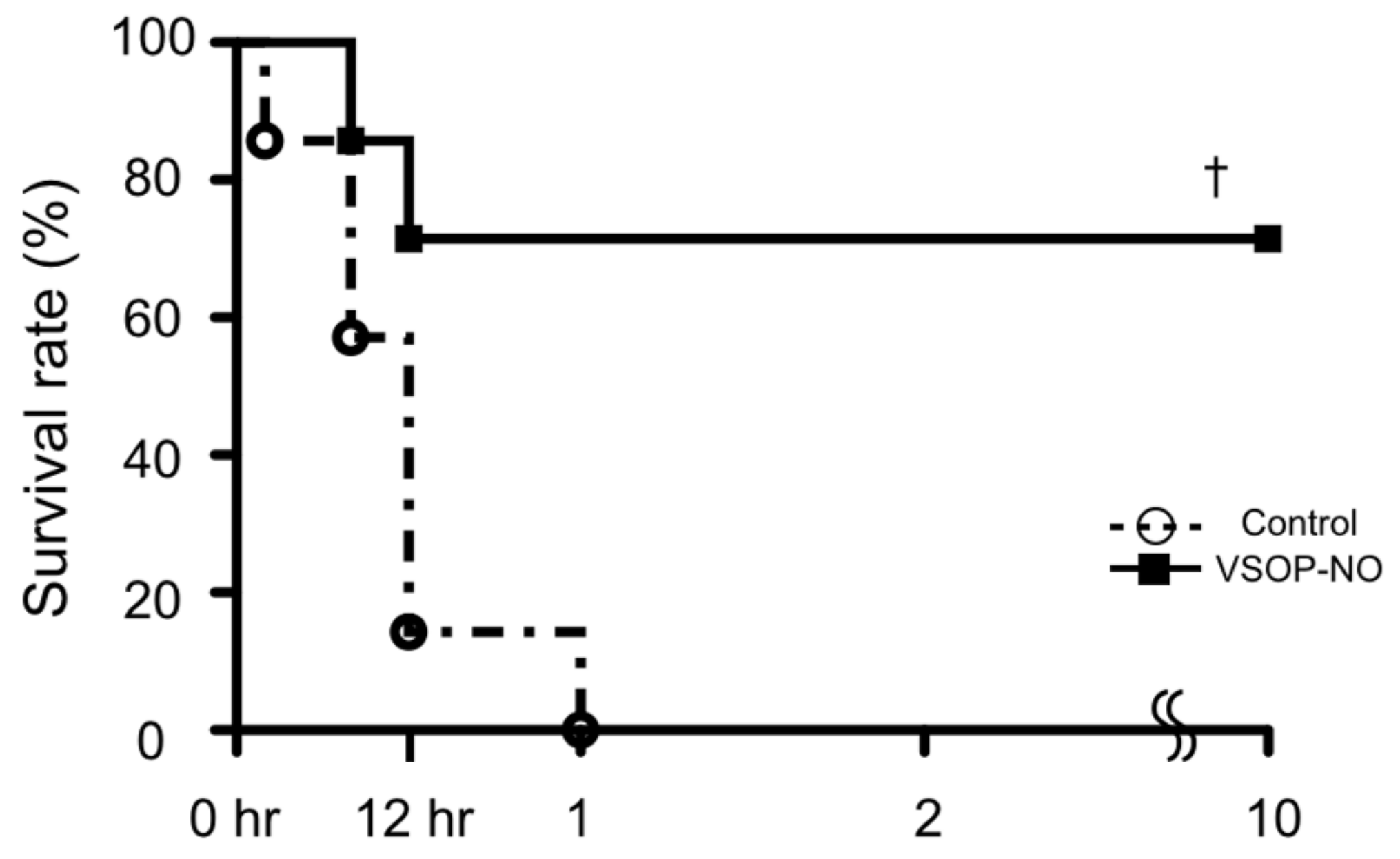

Numbers at risk

(days)

$\begin{array}{lllll}-\Theta-\text { Control } & 7 & 4 & 1 & 0 \\ - \text { - VSOP-NO } & 7 & 6 & 6 & 5\end{array}$

Figure 3 
A.
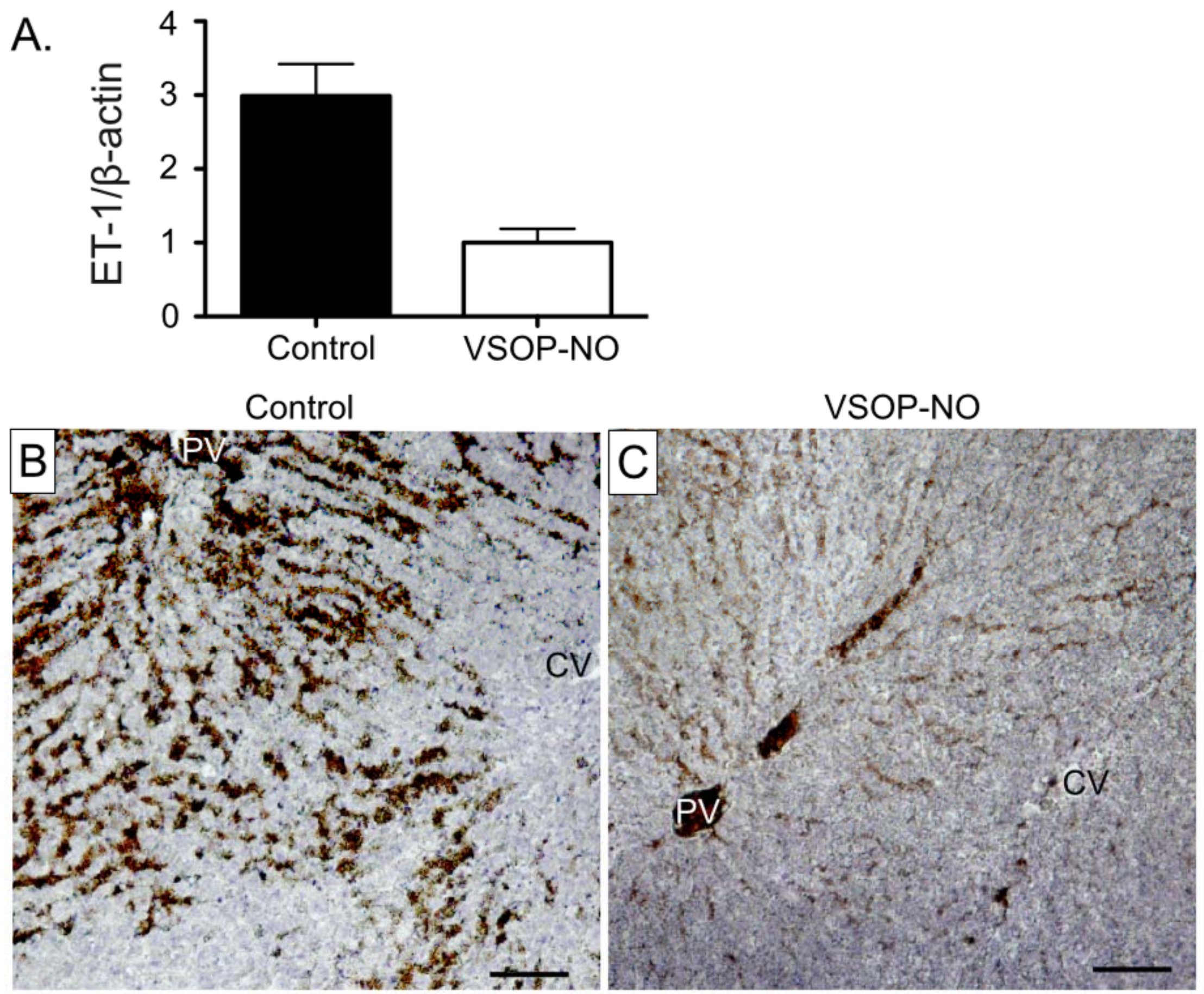

Figure 4 